\title{
Astrometry Missions: Probing the Galaxy and Beyond
}

\author{
F. Mignard \\ OCA/CERGA, av. Copernic 06130 Grasse (France)
}

\begin{abstract}
In the wake of the HIPPARCOS success four highly accurate space astrometry missions are now approved for launch within the next ten years. Stellar physics and galactic physics constitute the core science of these missions with deep impact on star formation and evolution, cosmic distance scales and age determination of the oldest stars. Summary data are provided in this paper to compare the goals and capabilities of these missions.
\end{abstract}

\section{Introduction}

The success of HIPPARCOS has demonstrated the scientific interest of accurate global astrometry in space and its major impact on many branches of astrophysics. It was clear that the potential of this technique had only been glimpsed with the 1 mas accuracy for a sample of rather bright stars $(V<9)$. Following this remarkable success several space astrometry missions have been studied aiming to explore the $\mu$ as world for much fainter sources. Four missions are now planned to be launched within the coming decade, two from the USA and two from Europe.

These new space astrometry missions share common features both in objectives and, to a lesser extent, in design. Regarding the data, several levels must be considered between the raw measurements and the final scientific output. Astrometric missions produce virtually no usable data until the mission is over and all the data sets are analyzed, which happens to be a very complex step.

Typically the direct results of a global space astrometry mission resulting from the raw measurements and the data processing are :

- Accurate positions, absolute parallaxes, proper motions for millions of stars.

- Multi-epochs photometry in several bands at the milli-magnitude level.

- Radial velocity

- Spectrophotometry

Not every mission is able to perform all this variety of observations. It depends on the instruments and detectors included in the payload, on the observation strategy and the on-board storage capacities.

Astrometric measurements are the baseline, in particular with the direct measure of the absolute parallaxes which appears with a high priority in the 
design criteria. Accurate measurement of relative parallax can be achieved with ground-based optical interferometers over narrow fields. However, when these measurements are converted to absolute parallaxes, the final accuracy is not better than about 1 mas because the average effect of the not-very-distant background reference stars is unknown. Though very high accuracy (10-20 $\mu \mathrm{as})$ measurements of absolute parallax are achieved in differential radio interferometry over small angles, both these measurements are limited to relatively small numbers of objects.

Photometry over the wide band in the visible can be conducted with the same instrument as astrometry. Dedicated filters of various widths are needed for more refined analysis of starlight, but at the expense of the number of photons available. Radial velocity is the third component of the motion needed for kinematical studies. It is a parallel acquisition only foreseen for the GAIA mission. It will fill a major gap, as the current surveys are very limited, both in number and in spectral coverage, compared to the size and quality of the astrometric or spectrophotometric surveys.

Whatever the interest of the direct output of an astrometric mission, the major support and main drive follow from the indirect results and applications to stellar and galactic physics. The list below bears witness to the extraordinary versatility of global astrometry which touches just about every aspect of astronomy. The major areas that benefit from space astrometry are primarily :

- Recalibration of the extragalactic distance scale (cepheids, RR Lyrae)

- Determination of the age of the globular clusters

- Determination of the absolute luminosities of a wide range of spectral types

- Detections of companion stars, brown dwarfs, and giant planets

- Detailed study of the structure, content and kinematics of our galaxy

- Materialization an optical inertial reference frame

- Mapping of the interstellar matter

- Contribution to fundamental physics (GR testing)

Each of the above items would be nearly sufficient to build a well balanced proposal for a space program, and in fact there are such proposals or even approved missions, like the French (and ESA supported) COROT. Probably the most awaited and easily publicized question that Space astrometry may answer is the detection of companion stars either in the substellar range $\left(<0.1 \mathcal{M}_{\odot}\right)$ or in the planetary range $\left(<10 M_{J}\right)$. An astrometric mission has the capability to survey a large number of potential systems over a wide range of spectral types and classes and produce valuable statistics on the formation rate of planetary systems and on their main characteristics. Although very appealing, I consider the impact on galactic and extragalactic physics as more significant with a recalibration of the extragalactic distance scale and a high resolution study of the Cepheid PL relation. No doubt that several populations of Cepheids will be discovered with slight variation with age, metallicity, location. Fundamental 
physics will also benefit from the astrometry through the accurate observation of the light bending in the solar gravitational field. Again HIPPARCOS has opened the way (Froeschlé et al., 1998) and demonstrated the possibility to use observations at wide angles from the Sun. Much higher accuracy is expected from the future missions.

\section{Main features of space astrometry missions}

Global astrometry missions belong to two broad categories according to the basic method used to survey the sky and to measure the positions of the stars.

One can survey in a systematic way all the sky with one or two fields of view with a continuous scanning, covering more or less uniformly the celestial sphere. This was the solution developed with much success for HIPPARCOS and it proved rather optimal, with each photon contributing with the same weight in the global adjustment of the astrometric parameters and in the realization of the reference system. Every object crossing the field of view and above a certain brightness threshold, depending on the instrument size and detector sensitivity, is recorded and ultimately appears in the final output with accurate astrometric position and motion given in a well defined inertial frame tied to extragalactic objects. Observing simultaneously in two widely separated viewing directions allows to carry out the instrument calibration and to construct a rigid network on the celestial sphere with only six degrees of freedom, that are frozen by including very distant extragalactic sources, virtually motionless.

Alternately, a pointing system can be used to look at a small number of predetermined targets, allowing to integrate the starlight over longer time according to the source brightness and/or scientific interest. The precision may then be much higher than with a scanning system which distributes the available observing time nearly uniformly over every source observable.

The two principles appear complementary and do not compete with each other. In addition it is difficult to imagine a system able to apply both principles within the same payload. The scanning missions rely on rigid and frozen scanning laws which determine completely the total time devoted to any sources, the epochs at which they are observed and the number of times they will cross the fields of view. An Input Catalogue can be used to define the observing program, otherwise one needs an on-board detection system. In this case the survey is completed up to the limit of detection of the measuring system and a variety of sources can be observed like stars, solar system objects, galaxies. The system is very efficient, with virtually no dead-time, but lacks flexibility and cannot work in an observatory mode. The sky coverage is systematic (Fig. 1), gradual as the mission progresses.

With a pointing mission, the basic design is not meant for a survey but just the opposite : careful and frequent observations of a small sample of preselected sources of high astrophysical interest. The integration time can be adapted (quickly ?) to any source and the whole program can be rescheduled to meet new and important requests. The price to pay is a significant dead time for the pointing acquisition and subsequent recalibrations. 


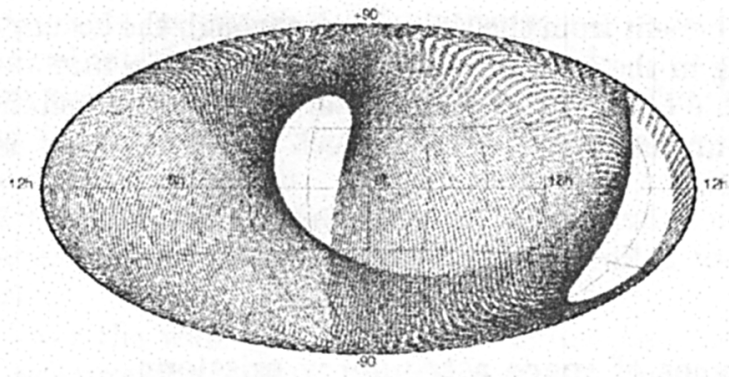

Figure 1. Typical sky coverage obtained with a scanning satellite designed for an astrometry mission. The plot corresponds to the GAIA scanning law over 60 days. Some areas have already been observed several times while others have not yet been visited.

\section{Comparison of the four approved missions}

At the moment there are four space missions which have been formally approved for a launch before 2012 .

The oldest one is the Space Interferometry Mission (SIM) developed by the Jet Propulsion Laboratory under contract with NASA and several industrial partners. SIM will be the first space mission to use optical interferometry and it will also pioneer a technique to block out (null) the light of bright stars to take images of the interesting areas close to the star.

The other three missions are FAME (Full-Sky Astrometric Mapping Explorer) a joint program between NASA and the USNO, the German mission DIVA (Double Interferometer for visual Astrometry or Deutsches Interferometer für Vielkanalphotometrie und Astrometrie), and GAIA recently selected in the science program of the European Space Agency.

The instrument and main mission parameters are listed in Table 1 for the four missions, with a comparison to the HIPPARCOS performances. The pioneering work of HIPPARCOS is somewhat dwarfed by these ambitious and far reaching projects that will benefit from the advance in technology (the main factors are the advent of large arrays of CCD chips and the on-board computing power) over twenty years.

As seen in Table 1 , the three survey missions belong to the scanning category while the only pointing mission (SIM) stands alone, with a very limited number of sources reached with an incredible accuracy. The other three missions will provide an excellent reference frame and improve dramatically the distance scale. Clearly DIVA and FAME constitute an intermediate step between HIPPARCOS and GAIA and have significant overlap.

- There are clearly two groups of mission appearing in Table 1 with two quick and relatively low-cost missions (DIVA and FAME) and two big, much more ambitious, expensive and to be developed over a longer timescale (GAIA and SIM). The first two build essentially on the HIPPARCOS concept using more advanced technology and their scientific program is an exten- 
Table 1. Summary data for the space astrometry missions. $V_{\text {lim }}$ is the limiting magnitude, $V_{\text {compl. }}$ is the magnitude of completion of the survey, $\mathrm{R}_{10 \%}$ is the distance at which the relative accuracy of the parallaxes is $10 \%$. The next to last entry refers to the use or the independence of an input catalogue. The last line indicates if radial velocities are measured.

\begin{tabular}{lccccc}
\hline Parameter & Hipparcos & DIVA & FAME & SIM & GAIA \\
\hline Type & Scanning & Scanning & Scanning & Pointing & Scanning \\
Launch & 1989 & $04 / 2004$ & $06 / 2004$ & 2009 & 2012 \\
Operations(yrs) & 3 & 2.5 & 5 & 4 & 5 \\
Results & 1996 & 2007 & $2007-09$ & & 2018 \\
$\mathrm{~V}_{\text {lim }}$ & 12 & $16(\mathrm{~K} 0)$ & 15 & 20 & 20 \\
$\mathrm{~V}_{\text {compl. }}$ & $7-9$ & 15 & 14 & - & 20 \\
$\sigma_{\pi}(\mu$ as $)$ & $1000(\mathrm{~V}<9)$ & $200(\mathrm{~V}=9)$ & $50(\mathrm{~V}<9)$ & $4(\mathrm{~V}<16)$ & $10(\mathrm{~V}<15)$ \\
$\sigma_{\mu}(\mu$ as $/ \mathrm{yr})$ & $1000(\mathrm{~V}<9)$ & $250(\mathrm{~V}=9)$ & $50(\mathrm{~V}<9)$ & 2 & $5(\mathrm{~V}<15)$ \\
$\mathrm{R}_{10 \%}(\mathrm{kpc})$ & 0.1 & 0.5 & $2(\mathrm{~V}<9)$ & 25 & $10(\mathrm{~V}<15)$ \\
Survey & $\mathrm{N}$ & $\mathrm{Y}$ & $\mathrm{Y}$ & $\mathrm{N}$ & $\mathrm{Y}$ \\
$\mathrm{N}$ Objects & $10^{5}$ & $3 \times 10^{7}$ & $4 \times 10^{7}$ & $2 \times 10^{4}$ & $10^{9}$ \\
Input Cat. & $\mathrm{Y}$ & $\mathrm{N}$ & $\mathrm{Y}$ & $\mathrm{Y}$ & $\mathrm{N}$ \\
$V_{r}$ & $\mathrm{~N}$ & $\mathrm{~N}$ & $\mathrm{~N}$ & $\mathrm{~N}$ & $\mathrm{Y}(\mathrm{V}<17)$ \\
\hline \hline
\end{tabular}

sion (deeper, better, cheaper) of the HIPPARCOS achievements. They go beyond HIPPARCOS in three respects: (1) precision is improved allowing to go farther with accurate parallaxes; (2) they do accurate photometry in several wavebands, enabling better characterization of stars; (3) they are primarily survey mission while the scientific output of HIPPARCOS was considerably limited by its using of a predetermined list of stars, implying a very small unbiased sample.

- The two small scanning missions (DIVA and FAME) are scheduled for an early launch in 2004 while for SIM and GAIA one will have to wait until the end of the decade. SIM is so challenging in term of technology (nanometer stabilization of the optical path, sub-nanometer laser metrology over separation of meters) and overall cost, that the practical implementation could take longer and be more expensive than expected today, even though the feasibility is considered as proven. So it would not be surprising that both GAIA and SIM have at the end a comparable schedule.

- The difference in content between a scanning mission and a pointing one is conspicuous, with several orders of magnitude between the size of the GAIA and SIM catalogue at mission completion, although they have comparable 
faint limiting magnitude. The number of objects selected for SIM in each class of stars (subdwarfs, Cepheids, RR Lyr, HGB) in different locations (Bulge, globular clusters, Magellanic clouds, etc) will be very limited, raising questions about the real impact of the mission in the understanding of the Galaxy and its capability to disentangle complex physical or dynamical processes. However the efficiency of the small angle astrometry for planetary detection and that of the imaging system for protoplanetary disks and compact galaxies are an asset undisputed by GAIA.

It is interesting to conclude this short presentation by showing in perspective the current and future ground based surveys and that to be carried out from space in the coming decade. This is shown in Fig. 2 for the position and parallaxes (left) and for the proper motion (right), by indicating for each program the size of the catalogue vs. its astrometric precision. The role of space astrometry is striking as it allows to produce very large catalogues (comparable in size to the largest surveys in the visible and infra-red) but with a precision which cannot be reached on the ground, in particular for the parallax. The global nature of the astrometry has also an advantage not visible on these plots regarding its homogeneity and its lack of systematic zonal errors, as it has been brightly demonstrated by HIPPARCOS.
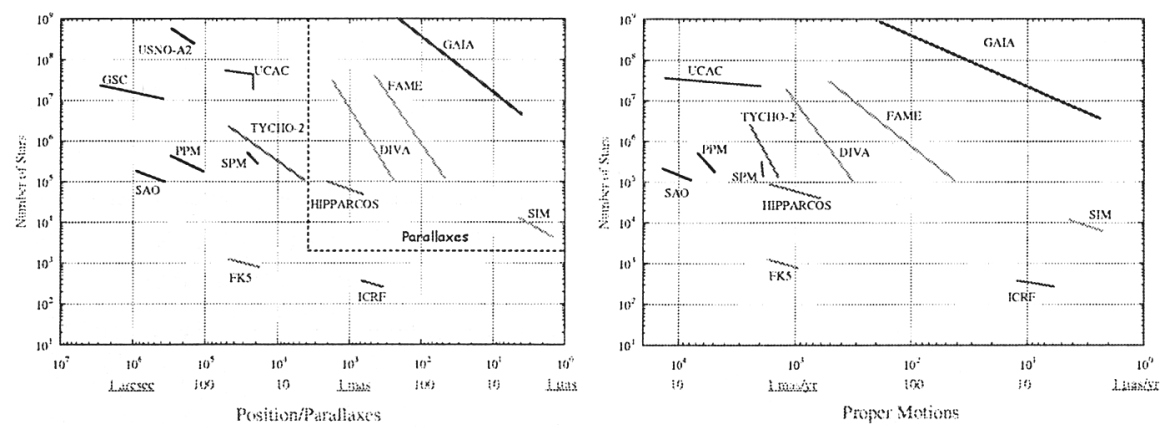

Figure 2. Comparison of recent and future stellar catalogues with astrometrical purposes. The impact of Hipparcos and that of the future space astrometry missions is clearly visible, both by the number of sources, the precision and the measurement of parallaxes

\section{References}

ESA 2000, GAIA, Composition, formation and evolution of the Galaxy, ESASCI $(2000) 4$.

Froeschlé M., Mignard F. \& Arenou F. 19978, proc. of the ESA Symp. 'Hipparcos - Venice 97 ', ESA SP-402, p. 49.

Roeser et al. 2000, DIVA : Vorschlag für eine Kleinmission an das DLR.

SIM 2000, The Space Interferometry Mission : Taking the Measure of the Universe. Final Report of the SIMSWG. 